MATHEMATICS OF COMPUTATION

Volume 72 , Number 241, Pages 335-354

S 0025-5718(01)01379-5

Article electronically published on July 22, 2001

\title{
MACRO-ELEMENTS AND STABLE LOCAL BASES FOR SPLINES ON POWELL-SABIN TRIANGULATIONS
}

\author{
MING-JUN LAI AND LARRY L. SCHUMAKER
}

\begin{abstract}
Macro-elements of arbitrary smoothness are constructed on Powell-Sabin triangle splits. These elements are useful for solving boundaryvalue problems and for interpolation of Hermite data. It is shown that they are optimal with respect to spline degree, and we believe they are also optimal with respect to the number of degrees of freedom. The construction provides local bases for certain superspline spaces defined over Powell-Sabin refinements. These bases are shown to be stable as a function of the smallest angle in the triangulation, which in turn implies that the associated spline spaces have optimal order approximation power.
\end{abstract}

\section{INTRODUCTION}

Let $\triangle$ be the triangulation of a polygonal domain $\Omega$ in $\mathbb{R}^{2}$. In this paper we are interested in polynomial spline spaces of the form

$$
\mathcal{S}_{d}^{r}(\triangle):=\left\{s \in C^{r}(\Omega):\left.s\right|_{T} \in \mathcal{P}_{d} \text { for all } T \in \triangle\right\},
$$

where $d>r \geq 0$ are given integers and $\mathcal{P}_{d}$ is the space of bivariate polynomials of degree $d$. A basis $\left\{B_{i}\right\}_{i=1}^{n}$ for a spline space $\mathcal{S}$ is called a stable local basis provided that there exist constants $\ell, K_{1}, K_{2}$ depending only on the smallest angle in $\triangle$ such that

1) for each $1 \leq i \leq n$, there is a vertex $v_{i}$ of $\triangle$ for which $\operatorname{supp}\left(B_{i}\right) \subseteq \operatorname{star}^{\ell}\left(v_{i}\right)$,

2) for all choices of the coefficient vector $c=\left(c_{1}, \ldots, c_{n}\right)$,

$$
K_{1}\|c\|_{\infty} \leq\left\|\sum_{i=1}^{n} c_{i} B_{i}\right\|_{\infty} \leq K_{2}\|c\|_{\infty}
$$

Here $\operatorname{star}^{0}(v)$ is defined to be the set of all triangles surrounding a vertex $v$, and $\operatorname{star}^{\ell}(v)$ is defined to be the union of $\operatorname{all} \operatorname{star}^{0}(w)$ where $w$ is a vertex of $\operatorname{star}^{\ell-1}(v)$.

It is known that if a space of splines $\mathcal{S}$ of degree $d$ contains $\mathcal{P}_{d}$ and has a stable local basis, then it provides optimal order approximations of smooth functions (see Remark 11.3). Such spaces are of particular importance in applications, including data fitting and the solution of boundary-value problems.

Finding stable local bases for spline spaces $\mathcal{S}_{d}^{r}(\triangle)$ is a nontrivial task for $r>0$, and for general triangulations can only be done when $d \geq 3 r+2$ (see Remark 11.4).

Received by the editor March 8, 2000 and, in revised form, January 31, 2001.

2000 Mathematics Subject Classification. Primary 41A15, 65M60, 65N30.

Key words and phrases. Macro-elements, stable bases, spline spaces.

The first author was supported by the National Science Foundation under grant DMS-9870187.

The second author was supported by the National Science Foundation under grant DMS9803340 and by the Army Research Office under grant DAAD-19-99-1-0160. 
The first constructions were for very special superspline subspaces of $\mathcal{S}_{d}^{r}(\triangle)$ and can be found in [2], [16]. A construction for arbitrary spline spaces $\mathcal{S}_{d}^{r}(\triangle)$ and corresponding superspline subspaces was discovered only very recently (see [3]).

To get stable bases for spline spaces with $d<3 r+2$, we have to restrict ourselves to classes of triangulations with a special structure. In this paper we work with Powell-Sabin triangulations $\triangle_{P S}$ which are obtained from an arbitrary triangulation $\triangle$ by splitting each triangle into six subtriangles (see Section 2). The main result of this paper is an explicit construction of stable local bases for certain superspline subspaces of $\mathcal{S}_{d(r)}^{r}\left(\triangle_{P S}\right)$, where

$$
d(r):= \begin{cases}(9 m+1) / 2, & \text { if } r=2 m \text { and } m \text { is odd, } \\ (9 m+2) / 2, & \text { if } r=2 m \text { and } m \text { is even, } \\ (9 m+4) / 2, & \text { if } r=2 m+1 \text { and } m \text { is even, } \\ (9 m+5) / 2, & \text { if } r=2 m+1 \text { and } m \text { is odd, }\end{cases}
$$

for all $r>1$. As a by-product of the construction, we obtain certain useful macroelements which can be used in the numerical solution of boundary-value problems and to solve Hermite interpolation problems. These elements are improvements on similar elements obtained earlier in [8], [20]-24] (see Section 9 for details).

The paper is organized as follows. In Section 2 we discuss Powell-Sabin refinements and in Section 3 the Bernstein-Bézier technique. In Sections 4 and 5 we treat the case $r=2 m$, while Sections 6 and 7 are devoted to the case $r=2 m+1$. In Section 8 we show how our constructions yield interesting new macro-elements for all choices of smoothness $r$, and in Section 9 we compare them with previously available macro-elements on Powell-Sabin splits. In Section 10 we show that our choices of degrees and supersmoothness are optimal in a certain sense. The last section is devoted to remarks.

\section{Powell-Sabin Refinements}

Definition 2.1. Given a triangulation $\triangle$ of a set $\Omega$, the Powell-Sabin refinement of $\triangle$ is the triangulation obtained as follows:

1) connect the incenter $v_{T}$ of each triangle $T$ in $\triangle$ to the three vertices of $T$;

2) connect incenters of neighboring triangles of $\triangle$ to each other;

3 ) if $T$ is a triangle with an edge $e$ on the boundary of $\Omega$, connect the incenter of $T$ to the center of $e$.

In the special case where this refinement process is applied to a single triangle $T:=\left\langle v_{1}, v_{2}, v_{3}\right\rangle$ with incenter $v$, we call the resulting Powell-Sabin refinement a Powell-Sabin cell. We denote such a cell by $\triangle_{v}$ and suppose that its boundary vertices are $\left\{v_{1}, w_{1}, v_{2}, w_{2}, v_{3}, w_{3}\right\}$ in counterclockwise order.

The following lemma shows that the Powell-Sabin refinement process is well defined and that the smallest angle in $\triangle_{P S}$ can be bounded below by a constant depending on the smallest angle in $\triangle$ (see also [23]).

Lemma 2.2. Suppose $\triangle_{P S}$ is the Powell-Sabin refinement of a given triangulation $\triangle$. Then $\theta_{P S} \geq \theta_{\triangle} \sin \left(\theta_{\triangle}\right) / 4$, where $\theta_{P S}$ is the smallest angle in $\triangle_{P S}$ and $\theta_{\triangle}$ is the smallest angle in $\triangle$. Moreover, for each edge $e:=\left\langle v_{1}, v_{2}\right\rangle$ of $\triangle$, the point $w_{1}$ obtained from step 2 of Definition 2.1 lies in the interior of $e$. More precisely, there exist constants $0<K_{1}<K_{2}$, depending only on the minimum $\theta_{\triangle}$ of the interior 


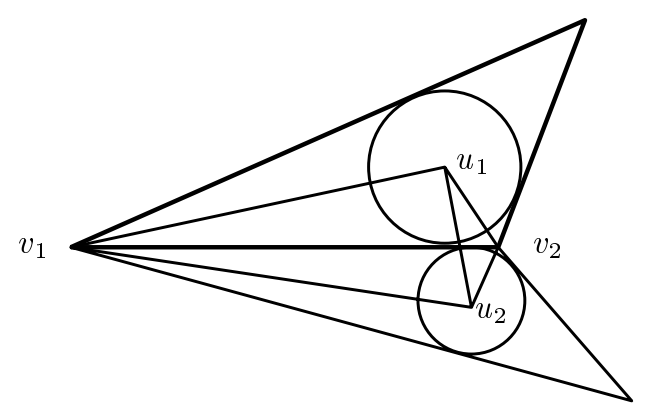

Figure 1. The geometry of incenters

angles of $\triangle$ and the exterior angles of the polygonal set $\Omega$ at boundary vertices of $\triangle$, such that

$$
0<K_{1} \leq \frac{h_{1}}{h_{2}} \leq K_{2},
$$

where $h_{1}=\left|\left\langle v_{1}, w_{1}\right\rangle\right|$ and $h_{2}=\left|\left\langle w_{1}, v_{2}\right\rangle\right|$.

Proof. We consider the case where $e:=\left\langle v_{1}, v_{2}\right\rangle$ is an edge between two triangles $T_{1}$ and $T_{2}$ (see Figure 1). The case where $e$ is a boundary edge of $\triangle$ and $w_{1}$ is its center is similar. Let $\theta$ be the minimum of the interior angles of $T_{1} \cup T_{2}$ and the exterior angles at $v_{1}$ and $v_{2}$. Suppose $u_{1}$ and $u_{2}$ are the incenters of $T_{1}$ and $T_{2}$. Let $\alpha_{1}$ and $\alpha_{2}$ be the angles at $v_{1}$ of $T_{1}$ and $T_{2}$, and $\tilde{\alpha}_{1}$ and $\tilde{\alpha}_{2}$ the angles at $v_{2}$. These angles are bounded below by $\theta / 2$. Consider the triangles $T:=\left\langle u_{1}, v_{1}, u_{2}\right\rangle$ and $\widetilde{T}:=\left\langle u_{1}, u_{2}, v_{2}\right\rangle$ with angles $\beta_{1}, \beta, \beta_{2}$ and $\tilde{\beta}_{1}, \tilde{\beta}_{2}, \tilde{\beta}$, where $\beta:=\left(\alpha_{1}+\alpha_{2}\right) / 2$ and $\tilde{\beta}:=\left(\tilde{\alpha}_{1}+\tilde{\alpha}_{2}\right) / 2$. It is easy to see that

$$
\theta \leq \beta, \tilde{\beta} \leq \pi-\theta
$$

Let $r_{1}:=\left|\left\langle v_{1}, u_{1}\right\rangle\right|, r_{2}:=\left|\left\langle v_{1}, u_{2}\right\rangle\right|, \tilde{r}_{1}:=\left|\left\langle v_{2}, u_{1}\right\rangle\right|, \tilde{r}_{2}:=\left|\left\langle v_{2}, u_{2}\right\rangle\right|, l_{1}:=\left|\left\langle u_{1}, w_{1}\right\rangle\right|$, and $l_{2}:=\left|\left\langle u_{2}, w_{1}\right\rangle\right|$. Then using the law of sines, we see that

$$
\begin{array}{ll}
\beta_{1} \geq \sin \left(\beta_{1}\right) \geq \frac{r_{2} \sin (\beta)}{l_{1}+l_{2}}, & \beta_{2} \geq \sin \left(\beta_{2}\right) \geq \frac{r_{1} \sin (\beta)}{l_{1}+l_{2}} \\
\tilde{\beta}_{1} \geq \sin \left(\tilde{\beta}_{1}\right) \geq \frac{\tilde{r}_{2} \sin (\tilde{\beta})}{l_{1}+l_{2}}, & \tilde{\beta}_{2} \geq \sin \left(\tilde{\beta}_{2}\right) \geq \frac{\tilde{r}_{1} \sin (\tilde{\beta})}{l_{1}+l_{2}} .
\end{array}
$$

We claim that the ratios $r_{i} /\left(l_{1}+l_{2}\right)$ and $\tilde{r}_{i} /\left(l_{1}+l_{2}\right)$ are bounded below by $\theta / 4$. Indeed, it is clear that the $r_{i}$ 's and $\tilde{r}_{i}$ 's are bounded below by the radius of the inscribed circles of $T_{1}$ and $T_{2}$, which in turn are bounded below by $\frac{|e|}{2} \tan (\theta) \geq$ $|e| \theta / 2$. Since the $l_{i}$ are bounded above by the length $|e|$ of $e$, we have

$$
r_{i} /\left(l_{1}+l_{2}\right) \geq \frac{|e| \theta}{2(|e|+|e|)}=\theta / 4,
$$

for $i=1,2$. Similar bounds hold for $\tilde{r}_{1}$ and $\tilde{r}_{2}$. It thus follows that the $\beta_{i}$ and $\tilde{\beta}_{i}$ are bounded below by $\theta \sin (\theta) / 4$. Now applying the law of sines again, we find that

$$
\frac{\sin \left(\beta_{1}\right)}{h_{1}}=\frac{\sin \left(\frac{\alpha_{1}}{2}\right)}{l_{1}}, \quad \frac{\sin \left(\tilde{\beta}_{1}\right)}{h_{2}}=\frac{\sin \left(\frac{\tilde{\alpha}_{1}}{2}\right)}{l_{1}} .
$$

Combining these facts leads to (2.1). 


\section{Constructing local Bases by the Bernstein-Bézier technique}

Our main tool for constructing stable local bases is the well-known BernsteinBézier technique used in most of the papers in our list of references. For any given $d$ and triangulation $\triangle$, let

$$
\mathcal{D}_{d, \triangle}:=\bigcup_{T \in \triangle} \mathcal{D}_{d, T}
$$

be the set of domain points, where

$$
\mathcal{D}_{d, T}:=\left\{\xi_{i j k}^{T}:=\frac{\left(i v_{1}+j v_{2}+k v_{3}\right)}{d}, i+j+k=d\right\}
$$

and $T:=\left\langle v_{1}, v_{2}, v_{3}\right\rangle$. As observed in [1], there is a 1-1 correspondence between

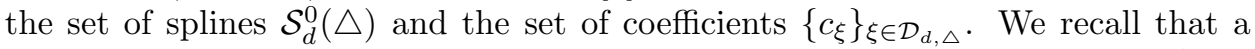
set $\mathcal{M} \subseteq \mathcal{D}_{d, \triangle}$ is a minimal determining set $(M D S)$ for a linear space $\mathcal{S} \subseteq \mathcal{S}_{d}^{0}(\triangle)$ provided that all coefficients of $s \in \mathcal{S}$ are uniquely determined from its coefficients $\left\{c_{\xi}\right\}_{\xi \in \mathcal{M}}$. If $\mathcal{M}$ is such a set, then there exists a corresponding set $\left\{B_{\xi}\right\}_{\xi \in \mathcal{M}}$ of dual splines satisfying

$$
\lambda_{\eta} B_{\xi}=\delta_{\xi, \eta}, \quad \text { all } \eta \in \mathcal{M}
$$

The splines $\left\{B_{\xi}\right\}_{\xi \in \mathcal{M}}$ are linearly independent and form a basis for $\mathcal{S}$. In general, considerable care is needed in choosing $\mathcal{S}$ and $\mathcal{M}$ to ensure that the dual basis is stable and local.

We now recall some standard notation concerning domain points. Given a triangle $T:=\left\langle v_{1}, v_{2}, v_{3}\right\rangle$, the ring of radius $n$ around $v_{1}$ is defined by

$$
R_{n}^{T}\left(v_{1}\right):=\left\{\xi_{i j k}^{T}: i=d-n\right\},
$$

and the disk of radius $n$ around $v_{1}$ is defined by

$$
D_{n}^{T}\left(v_{1}\right):=\left\{\xi_{i j k}^{T}: i \geq d-n\right\} .
$$

We have similar definitions at the other vertices of $T$. If $v$ is a vertex of a triangulation $\triangle$, we define

$$
\begin{aligned}
& R_{n}(v):=\bigcup R_{n}^{T}(v), \\
& D_{n}(v):=\bigcup D_{n}^{T}(v),
\end{aligned}
$$

where the union is taken over all triangles attached to $v$.

We close this section by introducing the class of supersplines of interest here. Given a triangulation $\triangle$ with vertices $\mathcal{V}$, let $\triangle_{P S}$ be its Powell-Sabin refinement. Let $\mathcal{W}$ be the set of incenters used to form the refinement, and let $\mathcal{E}$ be the set of edges $e$ of $\triangle_{P S}$ such that neither end of $e$ belongs to $\mathcal{V}$. These are the edges obtained by connecting incenters to other incenters, or to midpoints of boundary edges. Then given any integers $0 \leq r_{1}, r_{2}, r_{3}$, we define

$$
\begin{aligned}
& \mathcal{S}_{d}^{r_{1}, r_{2}, r_{3}}\left(\triangle_{P S}\right):=\left\{s \in \mathcal{S}_{d}^{r_{1}}\left(\triangle_{P S}\right): s \in C^{r_{2}}(v) \text { for all } v \in \mathcal{V},\right. \\
& \left.s \in C^{r_{3}}(v) \text { for all } v \in \mathcal{W}, \text { and } s \text { is } C^{r_{3}} \text { across all edges in } \mathcal{E}\right\} .
\end{aligned}
$$

Note that this is not a classical superspline space since here we have enforced additional continuity across certain (but not all) interior edges of $\triangle_{P S}$. 


\section{THE CASE $r=2 m$ WITH $m$ ODD}

In this section we work with the superspline spaces

$$
\mathcal{S}_{m}\left(\triangle_{P S}\right):=\mathcal{S}_{\frac{9 m+1}{2}}^{2 m, 3 m, 3 m}\left(\triangle_{P S}\right)
$$

To describe a minimal determining set whose corresponding set of dual splines form a stable local basis for $\mathcal{S}_{m}\left(\triangle_{P S}\right)$, we first examine the space $\mathcal{S}_{m}\left(\triangle_{v}\right)$ on the Powell-Sabin cell $\triangle_{v}$. Recall that we number the boundary vertices of $\triangle_{v}$ as $v_{1}, w_{1}, v_{2}, w_{2}, v_{3}, w_{3}$ and set $T^{[i]}:=\left\langle v, v_{i}, w_{i}\right\rangle$ for $i=1,2,3$.

Theorem 4.1. Let $\mathcal{M}$ be the union of the following sets of domain points:

1) $D_{3 m}^{T^{[i]}}\left(v_{i}\right)$ for $i=1,2,3$;

2) for $i=1,2,3$,
a) $\left\{\xi_{j, j-(3 m+3) / 2, d-2 j+(3 m+3) / 2}^{T^{[i]}}, \ldots, \xi_{j, 0, d-j}^{T^{[i]}}\right\}$ for $j=(3 m+3) / 2, \ldots, 2 m$,
b) $\left\{\xi_{j,(5 m-3) / 2-j, d-(5 m-3) / 2}^{T^{[i]}}, \ldots, \xi_{j, 0, d-j}^{T^{[i]}}\right\}$ for $j=2 m+1, \ldots,(5 m-3) / 2$,
c) $\xi_{j, d-j, 0}^{T[i]}$ for $j=3 m+1, \ldots,(7 m-1) / 2$;

3) $\xi_{d, 0,0}^{T^{[1]}}$.

Then $\mathcal{M}$ is a minimal determining set for $\mathcal{S}_{m}\left(\triangle_{v}\right)$, and the corresponding dual basis $\left\{B_{\xi}\right\}_{\xi \in \mathcal{M}}$ is a stable basis for $\mathcal{S}_{m}\left(\triangle_{v}\right)$. Moreover,

$$
\operatorname{dim} \mathcal{S}_{m}\left(\triangle_{v}\right)=\frac{57 m^{2}+54 m+13}{4}
$$

Proof. We first show that $\mathcal{M}$ is a minimal determining set. Suppose $s$ is a spline in $\mathcal{S}_{m}\left(\triangle_{v}\right)$ whose $B$-coefficients corresponding to points in $\mathcal{M}$ are set to prescribed values. We claim that all remaining coefficients are uniquely determined. First we observe that the coefficients corresponding to domain points in the disks $D_{3 m}\left(v_{i}\right)$ can be uniquely computed from those corresponding to domain points in item 1 by the classical smoothness conditions. This is a stable computation (cf. [16]) since by Lemma 2.2 the barycentric coordinate values entering into the smoothness conditions are bounded by a constant depending on the smallest angle in $\triangle_{v}$.

For each edge $e_{i}:=\left\langle v_{i}, v_{i+1}\right\rangle$ of $T$, we compute the coefficients associated with domain points in the rows $0, \ldots, 2 m$ parallel to $e_{i}$ by using the smoothness conditions across the edges $\left\langle v, w_{i}\right\rangle$. Then for each edge $e_{i}$, we compute coefficients corresponding to domain points in the rows $2 m+1, \ldots,(5 m-1) / 2$ parallel to $e_{i}$ by alternately using the smoothness conditions across the edges $\left\langle v, v_{i}\right\rangle$ and $\left\langle v, v_{i+1}\right\rangle$ (see Lemma 6.2 of [16]) and then across the edge $\left\langle v, w_{i}\right\rangle$. The lemma shows that this is a stable computation. At this point we have computed the coefficients of $s$ in the disks $D_{(7 m-1) / 2}\left(v_{i}\right)$.

To complete the proof, we note that by the supersmoothness at $v$, we can regard the coefficients of $s$ in the disk $D_{3 m}(v)$ as the coefficients of a polynomial $p$ of degree $3 m$ on the triangle $\widetilde{T}:=\left\langle u_{1}, u_{2}, u_{3}\right\rangle$, where $u_{i}:=\xi_{\frac{3 m+1}{2}}^{T^{[i]}} \frac{6 m}{2}, 0$ for $i=1,2,3$. These coefficients uniquely determine all of the derivatives $D_{x}^{\alpha} D_{y}^{\beta} p\left(u_{i}\right)$ for $0 \leq \alpha+\beta \leq$ $\frac{4 m-2}{2}$ and $i=1,2,3$. We now expand $p$ in terms of the Bernstein polynomials $\tilde{B}_{i j k}^{3 m}$ on the triangle $\widetilde{T}$. Using Lemma 2.2 , it follows that these derivatives uniquely and stably determine all of the $B$-coefficients $\tilde{c}_{i j k}$ of $p$ except for the coefficient $\tilde{c}_{m m m}$. 


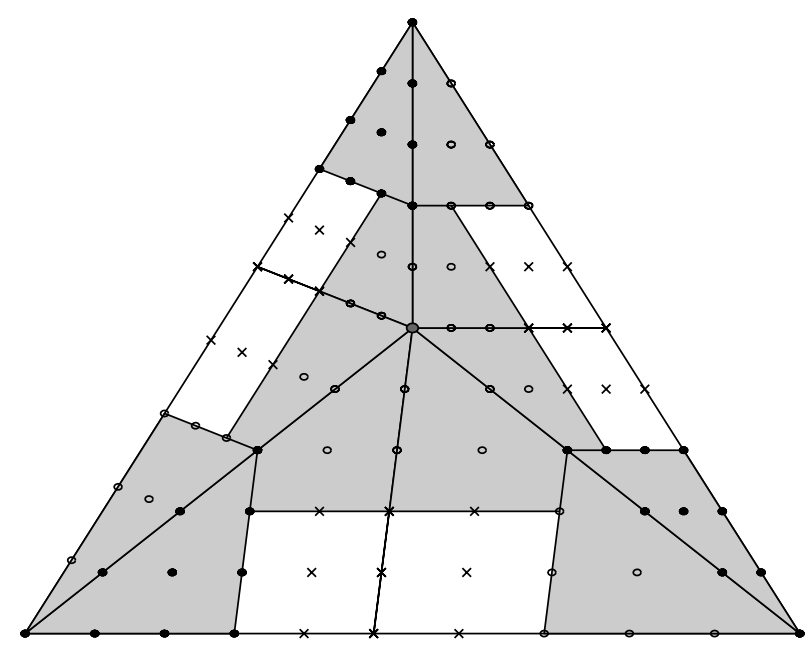

Figure 2. The macro-element $\mathcal{S}_{5}^{2,3,3}\left(\triangle_{v}\right)$

We compute this coefficient from the equation

$$
\tilde{c}_{m m m} \tilde{B}_{m m m}^{3 m}(v)=s(v)-\sum_{\substack{\nu+\mu+\kappa=3 m \\(\nu, \mu, \kappa) \neq(m, m, m)}} \tilde{c}_{\nu \mu \kappa} \tilde{B}_{\nu \mu \kappa}^{3 m}(v) .
$$

Note that $\tilde{B}_{m m m}^{3 m}(v)$ is bounded below by a constant depending on $\theta$ in view of Lemma 2.2 , and so $\tilde{c}_{m m m}$ can also be stably computed. We can now apply the de Casteljau algorithm (cf. [4], [5]) to subdivide the polynomial into a spline of degree $3 m$ on the Powell-Sabin split of $\widetilde{T}$. It is well known that this is a stable process. Finally, we transfer the computed coefficients back to the $B$-net for the spline $s$.

To compute the dimension of $\mathcal{S}_{m}\left(\triangle_{v}\right)$, we observe that

$$
\# \mathcal{M}=3\left[\left(\begin{array}{c}
3 m+2 \\
2
\end{array}\right)+\left(\begin{array}{c}
\frac{m-1}{2}+1 \\
2
\end{array}\right)+\left(\begin{array}{c}
\frac{m-3}{2}+1 \\
2
\end{array}\right)+\frac{m-1}{2}\right]+1,
$$

which reduces to the number in (4.1).

Example 4.2. The stable local MDS of Theorem 4.1 for $\mathcal{S}_{5}^{2,3,3}\left(\triangle_{v}\right)$ is shown in Figure 2. It contains 31 domain points. There are 10 points in each of the disks $D_{3}\left(v_{i}\right)$ (marked with dark circles), and one at the incenter (marked with a larger dark circle).

Discussion. We have shaded the disks $D_{3}\left(v_{i}\right)$ and $D_{3}(v)$. Once the points in the MDS are set, we use the $C^{3}$ smoothness conditions across the edges $\left\langle v, w_{i}\right\rangle$ to compute the coefficients marked with an $\mathrm{x}$. The remaining coefficients in $D_{3}(v)$ are then computed by the method described in the proof of Theorem 4.1.

Example 4.3. The stable local MDS of Theorem 4.1 for $\mathcal{S}_{14}^{6,9,9}\left(\triangle_{v}\right)$ is shown in Figure 3. It contains 172 domain points. There are 55 points in each of the disks $D_{9}\left(v_{i}\right)$ (marked with dark circles), and one at the incenter (marked with a larger dark circle). In addition, there are three points corresponding to item $2 \mathrm{c}$ of Theorem 4.1 (marked with diamonds), and three points corresponding to item $2 \mathrm{a}$ (marked with triangles). In this case there are no points corresponding to item $2 \mathrm{~b}$. 


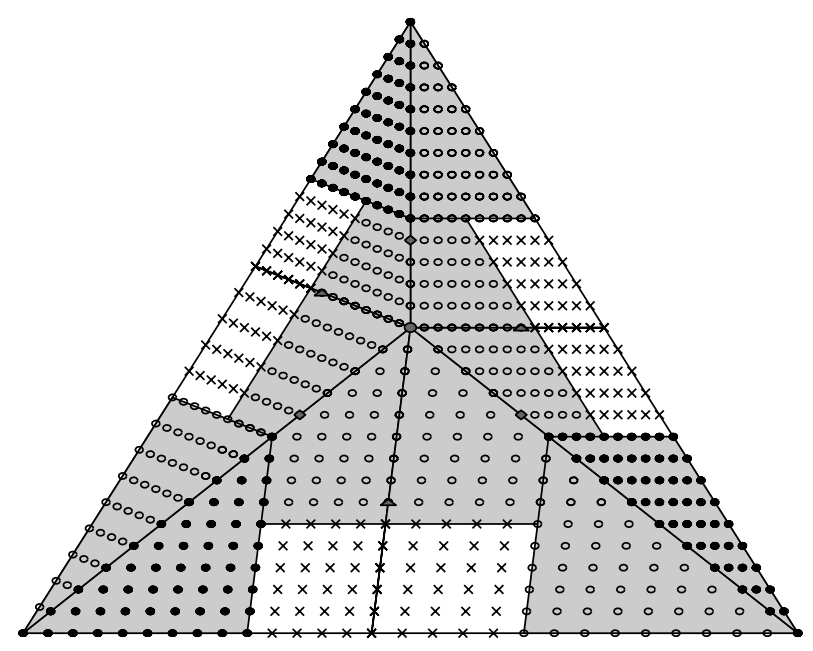

Figure 3 . The macro-element $\mathcal{S}_{14}^{6,9,9}\left(\triangle_{v}\right)$

Discussion. Here we have shaded the disks $D_{9}\left(v_{i}\right)$ and $D_{9}(v)$. Once the points in the MDS are set, we use the $C^{9}$ smoothness conditions across the edges $\left\langle v, w_{i}\right\rangle$ to compute the coefficients marked with an $\mathrm{x}$. The $C^{8}$ smoothness conditions across the edge $e_{i}:=\left\langle v, w_{i}\right\rangle$ are then used to compute the coefficients in the 6 -th row parallel to $e_{i}$. Using the smoothness conditions across the edges $\left\langle v, v_{i}\right\rangle$, we then compute the remaining coefficients on the rings $R_{10}\left(v_{i}\right)$ for $i=1,2,3$. The remaining coefficients in $D_{9}(v)$ are then computed by the method described in the proof of Theorem 4.1.

We can now use the construction of Theorem 4.1 to create a stable local basis for $\mathcal{S}_{m}(\triangle)$.

Theorem 4.4. Let $\mathcal{M}$ be the following set of domain points:

1) for each $v \in \mathcal{V}$, choose a triangle $T$ of $\triangle_{P S}$ attached to $v$ and include $D_{3 m}^{T}(v)$;

2) for each edge $e=\left\langle v_{1}, v_{2}\right\rangle$ of $\triangle$, let $w_{1}$ be the vertex of $\triangle_{P S}$ on $e$, and let $T=\left\langle v, v_{1}, w_{1}\right\rangle$ be a triangle of $\triangle_{P S}$ containing the edge $\left\langle v_{1}, w_{1}\right\rangle$. Then include the points

a) $\left\{\xi_{j, j-(3 m+3) / 2, d-2 j+(3 m+3) / 2}^{T}, \ldots, \xi_{j, 0, d-j}^{T}\right\}$ for $j=(3 m+3) / 2, \ldots, 2 m$,

b) $\left\{\xi_{j,(5 m-3) / 2-j, d-(5 m-3) / 2}^{T}, \ldots, \xi_{j, 0, d-j}^{T}\right\}$ for $j=2 m+1, \ldots,(5 m-3) / 2$,

c) $\xi_{j, d-j, 0}^{T}$ for $j=3 m+1, \ldots,(7 m-1) / 2$;

3) for each triangle $T$ in $\triangle$, include the domain point at its incenter.

Then $\mathcal{M}$ is a minimal determining set for $\mathcal{S}_{m}(\triangle)$, and the corresponding dual basis $\left\{B_{\xi}\right\}_{\xi \in \mathcal{M}}$ forms a stable star-supported basis for $\mathcal{S}_{m}(\triangle)$. Moreover,

$$
\operatorname{dim} \mathcal{S}_{m}(\triangle)=\left(\begin{array}{c}
3 m+2 \\
2
\end{array}\right) V+\left(\begin{array}{c}
\frac{m-1}{2}+1 \\
2
\end{array}\right) E+\left[3\left(\begin{array}{c}
\frac{m-3}{2}+1 \\
2
\end{array}\right)+\frac{(3 m-1)}{2}\right] N,
$$

where $V, E, N$ are the number of vertices, edges, and triangles in $\triangle$.

Proof. Following the arguments used in the proof of Theorem 4.1, it is easy to verify that $\mathcal{M}$ is a minimal determining set and that the construction of a dual basis can 
be carried out in a stable way. It is also easy to see that each dual basis spline has $\operatorname{support}$ on $\operatorname{star}(v)$ for some vertex $v$. To get the dimension, we simply count the number of points in $\mathcal{M}$.

\section{THE CASE $r=2 m$ WITH $m$ EVEN}

In this section we work with the superspline spaces

$$
\mathcal{S}_{m}\left(\triangle_{P S}\right):=\mathcal{S}_{\frac{9 m+2}{2}}^{2 m, 3 m+3 m+1}\left(\triangle_{P S}\right)
$$

We begin with a result for a Powell-Sabin cell $\triangle_{v}$.

Theorem 5.1. Let $\mathcal{M}$ be the union of the following sets of domain points:

1) $D_{3 m}^{T^{[i]}}\left(v_{i}\right)$ for $i=1,2,3$;

2) for $i=1,2,3$,
a) $\left\{\xi_{j, j-(3 m+2) / 2, d-2 j+(3 m+2) / 2}^{T^{[i]}}, \ldots, \xi_{j, 0, d-j}^{T^{[i]}}\right\}$ for $j=(3 m+2) / 2, \ldots, 2 m$,
b) $\left\{\xi_{j,(5 m-2) / 2-j, d-(5 m-2) / 2}^{T^{[i]}}, \ldots, \xi_{j, 0, d-j}^{T^{[i]}}\right\}$ for $j=2 m+1, \ldots,(5 m-2) / 2$,
c) $\xi_{j, d-j, 0}^{T^{[i]}}$ for $j=3 m+1, \ldots, 7 m / 2$.

Then $\mathcal{M}$ is a minimal determining set for $\mathcal{S}_{m}\left(\triangle_{v}\right)$, and the corresponding dual basis $\left\{B_{\xi}\right\}_{\xi \in \mathcal{M}}$ is a stable basis for $\mathcal{S}_{m}\left(\triangle_{v}\right)$. Moreover,

$$
\operatorname{dim} \mathcal{S}_{m}\left(\triangle_{v}\right)=\frac{57 m^{2}+60 m+12}{4}
$$

Proof. The proof that $\mathcal{M}$ is a minimal determining set follows along the same lines as in the proof of Theorem 4.1. In particular, setting all coefficients of a spline $s \in \mathcal{S}_{m}\left(\triangle_{v}\right)$ corresponding to $\mathcal{M}$, we can stably compute all coefficients in the disks $D_{3 m}\left(v_{i}\right)$ using the smoothness conditions. Then we use the smoothness conditions across the edges $\left\langle v, w_{i}\right\rangle$ and those across the edges $\left\langle v, v_{i}\right\rangle$ to stably compute all coefficients of $s$ in the rows $0, \ldots,(5 m-2) / 2$ parallel to edges of $T$ and in the disks $D_{7 m / 2}\left(v_{i}\right)$.

The computation of the remaining coefficients follows along the lines of the proof of Theorem 4.1, but is slightly different since the MDS $\mathcal{M}$ does not contain the point $\xi_{d, 0,0}^{T^{[1]}}$. We now regard the coefficients of $s$ in the disk $D_{3 m+1}(v)$ to be the coefficients of a polynomial $p$ of degree $3 m+1$ on the triangle $\widetilde{T}:=\left\langle u_{1}, u_{2}, u_{3}\right\rangle$ with $u_{i}:=\xi_{\frac{3 m}{2}, \frac{6 m+2}{2}, 0}^{T^{[i]}}$ for $i=1,2,3$. These coefficients uniquely determine $D_{x}^{\alpha} D_{y}^{\beta} p\left(u_{i}\right)$ for $0 \leq \alpha+\beta \leq 2 m$. These derivatives uniquely and stably determine all coefficients of $p$ in the expansion in terms of Bernstein polynomials $\tilde{B}_{i j k}^{3 m+1}$ on the triangle $\widetilde{T}$. We then apply the de Casteljau algorithm to subdivide $p$ into a spline of degree $3 m+1$ on the Powell-Sabin split of $\widetilde{T}$, and then transfer the computed coefficients back to the $B$-net for the spline $s$.

To compute the dimension of $\mathcal{S}_{m}\left(\triangle_{v}\right)$, we observe that

$$
\# \mathcal{M}=3\left[\left(\begin{array}{c}
3 m+2 \\
2
\end{array}\right)+\left(\begin{array}{c}
\frac{m}{2}+1 \\
2
\end{array}\right)+\left(\begin{array}{c}
\frac{m-2}{2}+1 \\
2
\end{array}\right)+\frac{m}{2}\right],
$$

which reduces to the number in (5.1).

Example 5.2. The stable local MDS for $\mathcal{S}_{10}^{4,6,7}\left(\triangle_{v}\right)$ of Theorem 5.1 is shown in Figure 4. It contains 90 domain points. There are 28 points in each of the disks $D_{6}\left(v_{i}\right)$ (marked with dark circles). In addition there are three points corresponding 


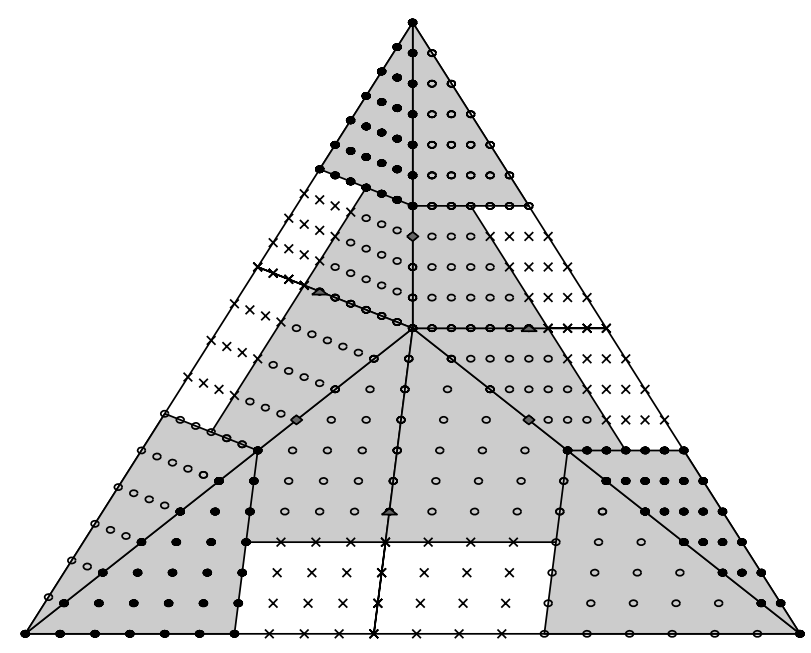

Figure 4. The macro-element $\mathcal{S}_{10}^{4,6,7}\left(\triangle_{v}\right)$

to item 2c (marked with diamonds), and three points corresponding to item $2 \mathrm{a}$ (marked with triangles).

Discussion. We have shaded the disks $D_{6}\left(v_{i}\right)$ and $D_{7}(v)$. Once the points in the MDS are set, we use the $C^{7}$ smoothness conditions across the edges $\left\langle v, w_{i}\right\rangle$ to compute the coefficients marked with an $\mathrm{x}$. The $C^{6}$ smoothness condition across the edges $\left\langle v, w_{i}\right\rangle$ are used to computed coefficients in the 4 -th rows parallel to the outer edges of $\triangle_{v}$. Then the smoothness conditions across the edges $\left\langle v, v_{i}\right\rangle$ are used to compute the remaining coefficients on the rings $R_{7}\left(v_{i}\right)$ for $i=1,2,3$. The remaining coefficients in $D_{7}(v)$ are then computed by the method described in the proof of Theorem 5.1.

Example 5.3. The stable local MDS for $\mathcal{S}_{19}^{8,12,13}\left(\triangle_{v}\right)$ of Theorem 5.1 is shown in Figure 5. It contains 291 domain points. There are 91 points in each of the disks $D_{12}\left(v_{i}\right)$ (marked with dark circles), In addition, there are six points corresponding to item 2c of Theorem 4.1 (marked with diamonds), nine points corresponding to item $2 \mathrm{a}$ (marked with triangles), and three points corresponding to item $2 \mathrm{~b}$ (marked with squares).

Discussion. We have shaded the disks $D_{12}\left(v_{i}\right)$ and $D_{13}(v)$. Once the points in the MDS are set, we use the $C^{13}$ smoothness conditions across the edges $\left\langle v, w_{i}\right\rangle$ to compute the coefficients marked with an $\mathrm{x}$. Smoothness conditions across $\left\langle v, w_{i}\right\rangle$ are also used to computed coefficients in rows 7 and 8 parallel to the outer edges $e_{i}$ of $T$. Then the $C^{8}$ smoothness conditions across the edges $\left\langle v, v_{i}\right\rangle$ are used to compute the remaining coefficients on the rings $R_{13}\left(v_{i}\right)$ for $i=1,2,3$. Then we compute coefficients in the 9 -th rows parallel to $e_{i}$, and finally the the remaining coefficients on the rings $R_{14}\left(v_{i}\right)$ for $i=1,2,3$. The remaining coefficients in $D_{19}(v)$ are then computed by the method described in the proof of Theorem 4.1.

We can now use the construction of Theorem 5.1 to create a stable local basis for $\mathcal{S}_{m}(\triangle)$. The following result is the analog of Theorem 4.4 and is proved in the same way. 


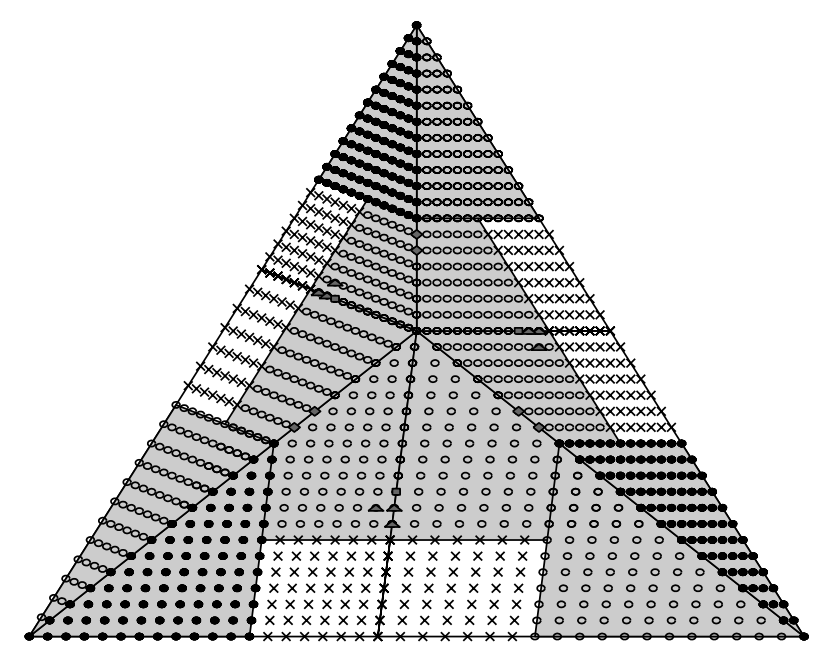

Figure 5. The macro-element $\mathcal{S}_{19}^{8,12,13}\left(\triangle_{v}\right)$

Theorem 5.4. Let $\mathcal{M}$ be the following set of domain points:

1) for each $v \in \mathcal{V}$, choose a triangle $T$ of $\triangle_{P S}$ attached to $v$ and include $D_{3 m}^{T}(v)$;

2) for each edge $e=\left\langle v_{1}, v_{2}\right\rangle$ of $\triangle$, let $w_{1}$ be the vertex of $\triangle_{P S}$ on e, and let $T=\left\langle v, v_{1}, w_{1}\right\rangle$ be a triangle of $\triangle_{P S}$ containing the edge $\left\langle v_{1}, w_{1}\right\rangle$. Then include the points
a) $\left\{\xi_{j, j-(3 m+2) / 2-j, d-2 j+(3 m+2) / 2}^{T}, \ldots, \xi_{j, 0, d-j}^{T}\right\}$ for $j=(3 m+2) / 2, \ldots, 2 m$,
b) $\left\{\xi_{j,(5 m-2) / 2-j, d-(5 m-2) / 2}^{T}, \ldots, \xi_{j, 0, d-j}^{T}\right\}$ for $j=2 m+1, \ldots,(5 m-2) / 2$,
c) $\xi_{j, d-j, 0}^{T}$ for $j=3 m+1, \ldots, 7 m / 2$.

Then $\mathcal{M}$ is a minimal determining set for $\mathcal{S}_{m}(\triangle)$, and the corresponding dual basis $\left\{B_{\xi}\right\}_{\xi \in \mathcal{M}}$ forms a stable star-supported basis for $\mathcal{S}_{m}(\triangle)$. Moreover,

$$
\operatorname{dim} \mathcal{S}_{m}(\triangle)=\left(\begin{array}{c}
3 m+2 \\
2
\end{array}\right) V+\left(\begin{array}{c}
\frac{m}{2}+1 \\
2
\end{array}\right) E+3\left[\left(\begin{array}{c}
\frac{m-2}{2}+1 \\
2
\end{array}\right)+\frac{m}{2}\right] N .
$$

\section{The CASE $r=2 m+1$ With $m$ EVEN}

In this section we work with the superspline spaces

$$
\mathcal{S}_{m}\left(\triangle_{P S}\right):=\mathcal{S}_{\frac{9 m+4}{2}}^{2 m+1,3 m+1,3 m+1}\left(\triangle_{P S}\right) .
$$

First we consider the case of a Powell-Sabin cell $\triangle_{v}$.

Theorem 6.1. Let $\mathcal{M}$ be the union of the following sets of domain points:

1) $D_{3 m+1}^{T^{[i]}}\left(v_{i}\right)$ for $i=1,2,3$;

2) for $i=1,2,3$,
a) $\left\{\xi_{j, j-(3 m+4) / 2, d-2 j+(3 m+4) / 4}^{T^{[i]}}, \ldots, \xi_{j, 0, d-j}^{T^{[i]}}\right\}$ for $j=(3 m+4) / 2, \ldots$, $2 m+1$,
b) $\left\{\xi_{j, 5 m / 2-j, d-5 m / 2}^{T^{[i]}}, \ldots, \xi_{j, 0, d-j}^{T^{[i]}}\right\}$ for $j=2 m+2, \ldots, 5 m / 2$. 
Then $\mathcal{M}$ is a minimal determining set for $\mathcal{S}_{m}\left(\triangle_{v}\right)$, and the corresponding dual basis $\left\{B_{\xi}\right\}_{\xi \in \mathcal{M}}$ is a stable basis for $\mathcal{S}_{m}\left(\triangle_{v}\right)$. Moreover,

$$
\operatorname{dim} \mathcal{S}_{m}\left(\triangle_{v}\right)=\frac{57 m^{2}+90 m+36}{4} .
$$

Proof. Setting all coefficients of a spline $s \in \mathcal{S}_{m}\left(\triangle_{v}\right)$ and using the smoothness conditions, we can stably compute all coefficients in the disks $D_{3 m+1}\left(v_{i}\right)$. Then we use the smoothness conditions across the edges $\left\langle v, w_{i}\right\rangle$ and those across the edges $\left\langle v, v_{i}\right\rangle$ to stably compute all coefficients of $s$ in the rows $0, \ldots, 5 \mathrm{~m} / 2$ parallel to edges of $T$ and in the disks $D_{(7 m+2) / 2}\left(v_{i}\right)$.

The computation of the remaining coefficients is similar to that in the proof of Theorem 5.1. We regard the coefficients of $s$ in the disk $D_{3 m+1}(v)$ to be the coefficients of a polynomial $p$ of degree $3 m+1$ on the triangle $\widetilde{T}:=\left\langle u_{1}, u_{2}, u_{3}\right\rangle$ with $u_{i}:=\xi_{\frac{3 m+2}{2}}^{T^{[i]}}, \frac{6 m+2}{2}, 0$ for $i=1,2,3$. These coefficients uniquely determine all of the derivatives $D_{x}^{\alpha} D_{y}^{\beta} p\left(u_{i}\right)$ for $0 \leq \alpha+\beta \leq 2 m$. These derivatives uniquely and stably determine all $B$-coefficients of $p$ in terms of Bernstein polynomials $\tilde{B}_{i j k}^{3 m+1}$ on the triangle $\widetilde{T}$. We then apply the de Casteljau algorithm to subdivide $p$ into a spline of degree $3 m+1$ on the Powell-Sabin split of $\widetilde{T}$, and then transfer the computed coefficients back to the $B$-net for the spline $s$.

The dimension of $\mathcal{S}_{m}\left(\triangle_{v}\right)$ is given by

$$
\# \mathcal{M}=3\left[\left(\begin{array}{c}
3 m+3 \\
2
\end{array}\right)+\left(\begin{array}{c}
\frac{m}{2}+1 \\
2
\end{array}\right)+\left(\begin{array}{c}
\frac{m-2}{2}+1 \\
2
\end{array}\right)\right]
$$

which reduces to the number in (6.1).

Example 6.2. The stable local MDS of Theorem 6.1 for $\mathcal{S}_{11}^{5,7,7}\left(\triangle_{v}\right)$ is shown in Figure 6 . It contains 111 domain points. There are 36 points in each of the disks $D_{6}\left(v_{i}\right)$ (marked with dark circles). In addition, there are three points corresponding to item 2a (marked with triangles).

Discussion. We have shaded the disks $D_{7}\left(v_{i}\right)$ and $D_{7}(v)$. Once the points in the MDS are set, we use the smoothness conditions across the edges $\left\langle v, w_{i}\right\rangle$ to compute the coefficients marked with an $\mathrm{x}$ and the coefficients in the 5 -th rows parallel to the outer edges of $\triangle_{v}$. Then the smoothness conditions across the edges $\left\langle v, v_{i}\right\rangle$ are used to compute the remaining coefficients on the rings $R_{8}\left(v_{i}\right)$. Finally, the remaining coefficients in $D_{7}(v)$ are computed by the method described in the proof of Theorem 6.1.

Example 6.3. The stable local MDS of Theorem 6.1 for $\mathcal{S}_{20}^{9,13,13}\left(\triangle_{v}\right)$ is shown in Figure 7. It contains 327 domain points. There are 105 points in each of the disks $D_{13}\left(v_{i}\right)$ (marked with dark circles). In addition, there are nine points corresponding to item 2a (marked with triangles), and three points corresponding to item $2 \mathrm{~b}$ (marked with squares).

Discussion. We have shaded the disks $D_{13}\left(v_{i}\right)$ and $D_{13}(v)$. Once the points in the MDS are set, we use the $C^{13}$ smoothness conditions across the edges $\left\langle v, w_{i}\right\rangle$ to compute the coefficients marked with an $\mathrm{x}$. The smoothness conditions are then used to compute the coefficients in rows number 8, 9, 10 parallel to the outer edges of $\triangle_{v}$ and in the disks $D_{15}\left(v_{i}\right)$. The remaining coefficients in $D_{7}(v)$ are then computed by the method described in the proof of Theorem 6.1 . 


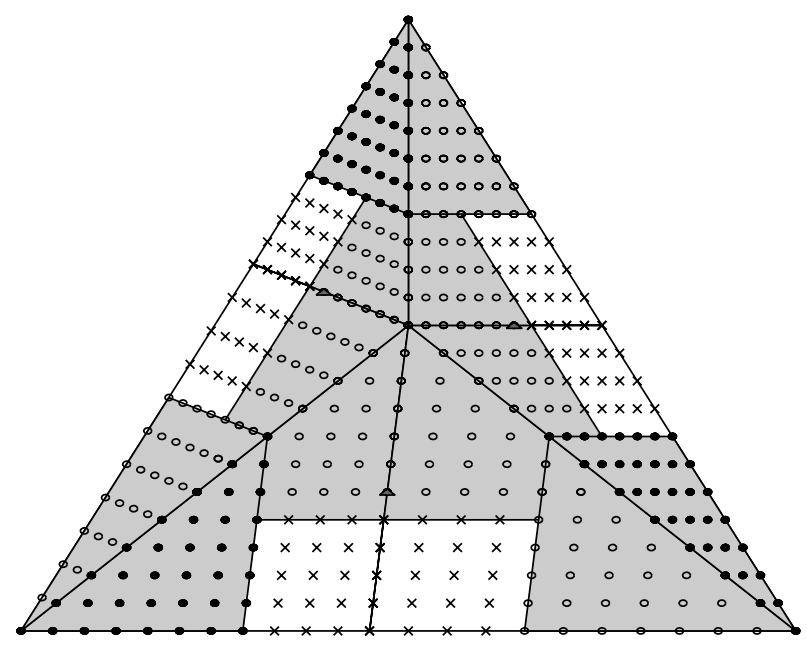

FiguRE 6 . The macro-element $\mathcal{S}_{11}^{5,7,7}\left(\triangle_{v}\right)$

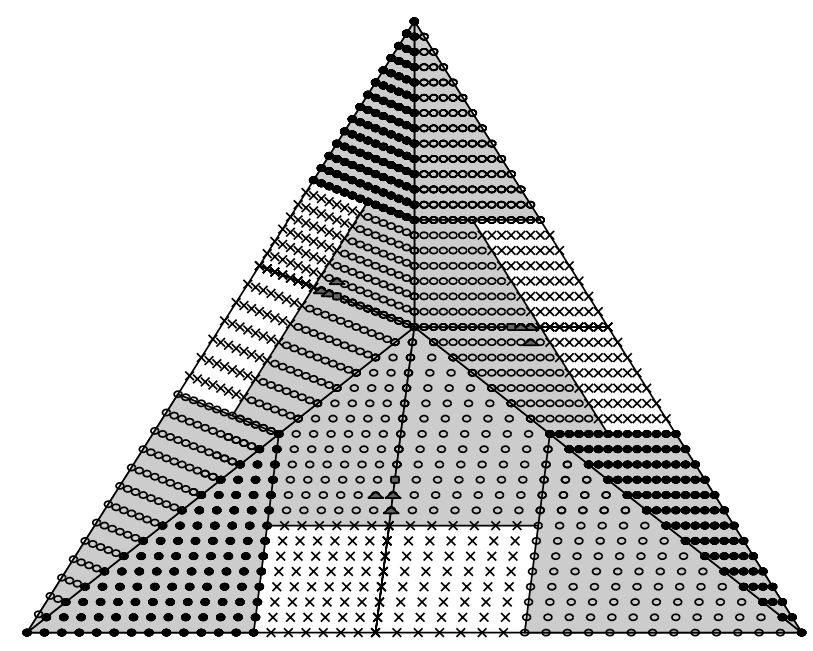

Figure 7. The macro-element $\mathcal{S}_{20}^{9,13,13}\left(\triangle_{v}\right)$

Theorem 6.4. Let $\mathcal{M}$ be the following set of domain points:

1) for each $v \in \mathcal{V}$, choose a triangle $T$ of $\triangle_{P S}$ attached to $v$ and include $D_{3 m+1}^{T}(v)$

2) for each edge $e=\left\langle v_{1}, v_{2}\right\rangle$ of $\triangle$, let $w_{1}$ be the vertex of $\triangle_{P S}$ on $e$, and let $T=\left\langle v, v_{1}, w_{1}\right\rangle$ be a triangle of $\triangle_{P S}$ containing the edge $\left\langle v_{1}, w_{1}\right\rangle$. Then include the points

a) $\left\{\xi_{j, j-(3 m+4) / 2, d-2 j+(3 m+4) / 4}^{T}, \ldots, \xi_{j, 0, d-j}^{T}\right\}$ for $j=(3 m+4) / 2, \ldots$, $2 m+1$,

b) $\left\{\xi_{j, 5 m / 2-j, d-5 m / 2}^{T}, \ldots, \xi_{j, 0, d-j}^{T}\right\}$ for $j=2 m+2, \ldots, 5 m / 2$. 
Then $\mathcal{M}$ is a minimal determining set for $\mathcal{S}_{m}(\triangle)$, and the corresponding dual basis $\left\{B_{\xi}\right\}_{\xi \in \mathcal{M}}$ forms a stable star-supported basis for $\mathcal{S}_{m}(\triangle)$. Moreover,

$$
\operatorname{dim} \mathcal{S}_{m}(\triangle)=\left(\begin{array}{c}
3 m+3 \\
2
\end{array}\right) V+\left(\begin{array}{c}
\frac{m}{2}+1 \\
2
\end{array}\right) E+3\left(\begin{array}{c}
\frac{m-2}{2}+1 \\
2
\end{array}\right) N
$$

7. ThE CASE $r=2 m+1$ WITH $m$ ODD

In this section we work with the superspline spaces

$$
\mathcal{S}_{m}\left(\triangle_{P S}\right):=\mathcal{S}_{\frac{9 m+5}{2}}^{2 m+1,3 m+1,3 m+2}\left(\triangle_{P S}\right) .
$$

We begin by examining a Powell-Sabin cell $\triangle_{v}$.

Theorem 7.1. Let $\mathcal{M}$ be the union of the following sets of domain points:

1) $D_{3 m+1}^{T^{[i]}}\left(v_{i}\right)$ for $i=1,2,3$;

2) for $i=1,2,3$,
a) $\left\{\xi_{j, j-(3 m+3) / 2, d-2 j+(3 m+3) / 2}^{T^{[i]}}, \ldots, \xi_{j, 0, d-j}^{T^{[i]}}\right\}$ for $j=(3 m+3) / 2, \ldots$, $2 m+1$,
b) $\left\{\xi_{j,(5 m+1) / 2-j, d-(5 m+1) / 2}^{T^{[i]}}, \ldots, \xi_{j, 0, d-j}^{T^{[i]}}\right\}$ for $j=2 m+2, \ldots,(5 m+1) / 2$.

Then $\mathcal{M}$ is a minimal determining set for $\mathcal{S}_{m}\left(\triangle_{v}\right)$, and the corresponding dual basis $\left\{B_{\xi}\right\}_{\xi \in \mathcal{M}}$ is a stable basis for $\mathcal{S}_{m}\left(\triangle_{v}\right)$. Moreover,

$$
\operatorname{dim} \mathcal{S}_{m}\left(\triangle_{v}\right)=\frac{57 m^{2}+96 m+39}{4}
$$

Proof. Setting all coefficients of a spline $s \in \mathcal{S}_{m}\left(\triangle_{v}\right)$ and using the smoothness conditions, we can stably compute all coefficients in the disks $D_{3 m+1}\left(v_{i}\right)$. Then we use the smoothness conditions across the edges $\left\langle v, w_{i}\right\rangle$ and those across the edges $\left\langle v, v_{i}\right\rangle$ to stably compute all coefficients of $s$ in the rows $0, \ldots,(5 m+1) / 2$ parallel to edges of $T$ and in the disks $D_{(7 m+3) / 2}\left(v_{i}\right)$.

The computation of the remaining coefficients follows in the same way as in the proof of Theorem 5.1. We regard the coefficients of $s$ in the disk $D_{3 m+2}(v)$ as the coefficients of a polynomial $p$ of degree $3 m+2$ on the triangle $\widetilde{T}:=\left\langle u_{1}, u_{2}, u_{3}\right\rangle$ with $u_{i}:=\xi_{\frac{3 m+1}{2}}^{T^{[i]}}, \frac{6 m+4}{2}, 0$ for $i=1,2,3$. These coefficients uniquely determine all of the derivatives $D_{x}^{\alpha} D_{y}^{\beta} p\left(u_{i}\right)$ for $0 \leq \alpha+\beta \leq 2 m+1$. We now expand $p$ in terms of the Bernstein polynomials $\tilde{B}_{i j k}^{3 m+2}$ on the triangle $\widetilde{T}$. These derivatives uniquely and stably determine all of the $B$-coefficients $\tilde{c}_{i j k}$ of $p$. We then apply the de Casteljau algorithm to subdivide the polynomial into a spline of degree $3 m+2$ on the PowellSabin split of $\widetilde{T}$, and then transfer the computed coefficients back to the $B$-net for the spline $s$.

The dimension of $\mathcal{S}_{m}\left(\triangle_{v}\right)$ is given by

$$
\# \mathcal{M}=3\left[\left(\begin{array}{c}
3 m+3 \\
2
\end{array}\right)+\left(\begin{array}{c}
\frac{m+1}{2}+1 \\
2
\end{array}\right)+\left(\begin{array}{c}
\frac{m-1}{2}+1 \\
2
\end{array}\right)\right]
$$

which reduces to the number in (7.1).

Example 7.2. The stable local MDS of Theorem 7.1 for $\mathcal{S}_{7}^{3,4,5}\left(\triangle_{v}\right)$ is shown in Figure 8. It contains 48 domain points. There are 15 points in each of the disks $D_{4}\left(v_{i}\right)$ (marked with dark circles). In addition there are three points corresponding to item 2a (marked with triangles). 


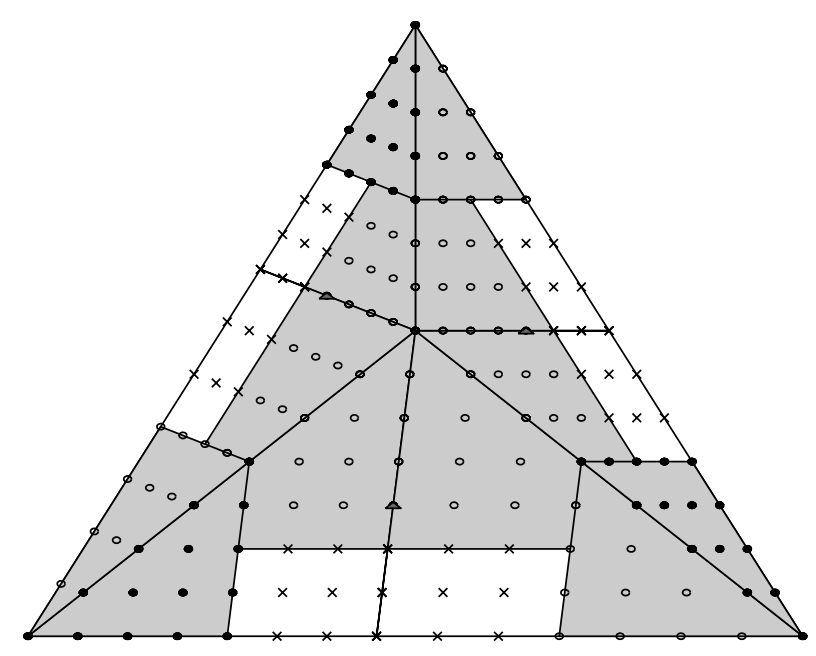

FiguRE 8 . The macro-element $\mathcal{S}_{7}^{3,4,5}\left(\triangle_{v}\right)$

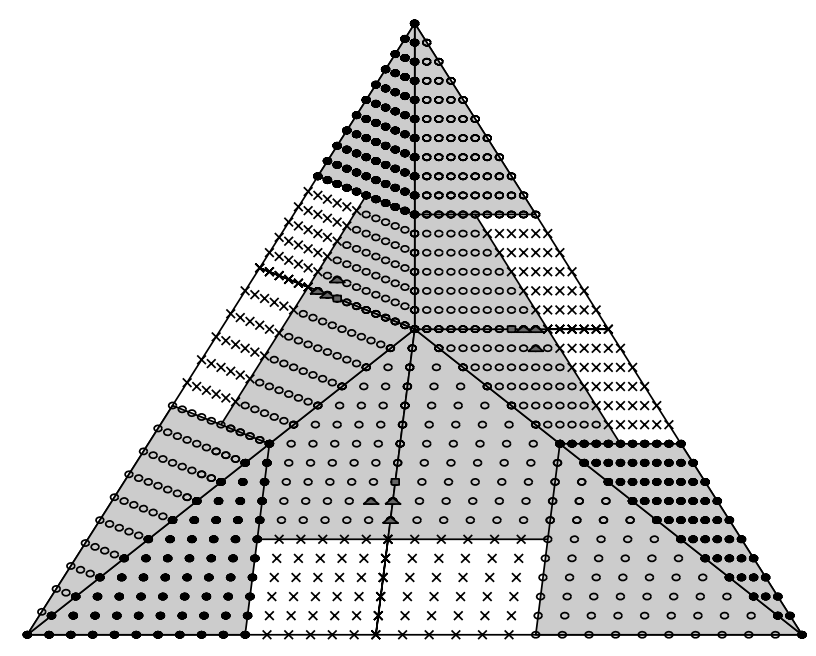

Figure 9. The macro-element $\mathcal{S}_{16}^{7,10,11}\left(\triangle_{v}\right)$

Discussion. We have shaded the disks $D_{4}\left(v_{i}\right)$ and $D_{5}(v)$. Once the points in the MDS are set, we use the $C^{5}$ smoothness conditions across the edges $\left\langle v, w_{i}\right\rangle$ to compute the coefficients marked with an $\mathrm{x}$. The $C^{4}$ smoothness condition across the edges $\left\langle v, w_{i}\right\rangle$ are used to computed coefficients in the 3-rd rows parallel to the outer edges of $\triangle_{v}$. Then we use the smoothness conditions across the edges $\left\langle v, v_{i}\right\rangle$ to determine the remaining coefficients on the rings $R_{5}\left(v_{i}\right)$. Finally, the remaining coefficients in $D_{5}(v)$ are then computed by the method described in the proof of Theorem 7.1.

Example 7.3. The stable local MDS of Theorem 7.1 for $\mathcal{S}_{16}^{7,10,11}\left(\triangle_{v}\right)$ is shown in Figure 9. It contains 210 domain points. There are 66 points in each of the disks 
$D_{10}\left(v_{i}\right)$ (marked with dark circles). In addition, there are nine points corresponding to item 2a (marked with triangles), and three points corresponding to item $2 \mathrm{~b}$ (marked with squares).

Discussion. We have shaded the disks $D_{10}\left(v_{i}\right)$ and $D_{11}(v)$. The smoothness conditions are used to determine all coefficients in the first 8 rows parallel to the outer edges of $T$ and in the disks $D_{12}\left(v_{i}\right)$. The remaining coefficients in $D_{11}(v)$ are then computed by the method described in the proof of Theorem 7.1.

Theorem 7.4. Let $\mathcal{M}$ be the following set of domain points:

1) for each $v \in \mathcal{V}$, choose a triangle $T$ of $\triangle_{P S}$ attached to $v$ and include $D_{3 m+1}^{T}(v)$

2) for each edge $e=\left\langle v_{1}, v_{2}\right\rangle$ of $\triangle$, let $w_{1}$ be the vertex of $\triangle_{P S}$ on e, and let $T=\left\langle v, v_{1}, w_{1}\right\rangle$ be a triangle of $\triangle_{P S}$ including the edge $\left\langle v_{1}, w_{1}\right\rangle$. Then include the points

a) $\left\{\xi_{j, j-(3 m+3) / 2, d-2 j+(3 m+3) / 2}^{T}, \ldots, \xi_{j, 0, d-j}^{T}\right\}$ for $j=(3 m+3) / 2, \ldots$, $2 m+1$,

b) $\left\{\xi_{j,(5 m+1) / 2-j, d-(5 m+1) / 2}^{T}, \ldots, \xi_{j, 0, d-j}^{T}\right\}$ for $j=2 m+2, \ldots,(5 m+1) / 2$.

Then $\mathcal{M}$ is a minimal determining set for $\mathcal{S}_{m}(\triangle)$, and the corresponding dual basis $\left\{B_{\xi}\right\}_{\xi \in \mathcal{M}}$ forms a stable star-supported basis for $\mathcal{S}_{m}(\triangle)$. Moreover,

$$
\operatorname{dim} \mathcal{S}_{m}(\triangle)=\left(\begin{array}{c}
3 m+3 \\
2
\end{array}\right) V+\left(\begin{array}{c}
\frac{m+1}{2}+1 \\
2
\end{array}\right) E+3\left(\begin{array}{c}
\frac{m-1}{2}+1 \\
2
\end{array}\right) N
$$

\section{MACRO-ELEMENTS}

The constructions of minimal determining sets for superspline spaces $\mathcal{S}_{m}\left(\triangle_{v}\right)$ on the Powell-Sabin split $\triangle_{v}$ of a single triangle $T$ given in Theorems 4.1, 5.1, 6.1 , and 7.1 can all be regarded as defining macro-elements. In the finite-element literature, such macro-elements are typically defined in terms of nodal parameters, i.e., derivatives. Here we have described them in terms of minimal determining sets of $B$-coefficients, but it is easy to translate to derivatives. We give three examples. Given a triangle $T=\left\langle v_{1}, v_{2}, v_{3}\right\rangle$, let $\triangle_{v}$ be the corresponding Powell-Sabin cell with center $v$ and boundary vertices $\left\{v_{1}, w_{1}, v_{2}, w_{2}, v_{3}, w_{3}\right\}$ in counterclockwise order.

Example 8.1. Any element in the superspline space $\mathcal{S}_{2}^{1}\left(\triangle_{v}\right)$ of Theorem 6.1 with $m=0$ is uniquely defined by the the derivatives $\left\{D_{x}^{\alpha} D_{y}^{\beta} s\left(v_{i}\right)\right\}$ for $0 \leq \alpha+\beta \leq 1$ and $i=1,2,3$.

Proof. It is well known from the Bernstein-Bézier theory that specifying this set of derivatives for an $s \in \mathcal{S}_{2}^{1}\left(\triangle_{P S}\right)$ is equivalent to setting the $B$-coefficients of $s$ corresponding to the domain points in the disks $D_{1}\left(v_{i}\right), i=1,2,3$.

Given any point $u$ in $T$, let $D_{\langle u, v\rangle}$ denote the directional derivative in the direction from $u$ to $v$.

Example 8.2. Any element in the superspline space $\mathcal{S}_{7}^{3,4,5}\left(\triangle_{v}\right)$ of Theorem 7.1 with $m=1$ is uniquely defined by the following set of 48 data (cf. Example 7.2 and Figure 8):

1) the derivatives $\left\{D_{x}^{\alpha} D_{y}^{\beta} s\left(v_{i}\right)\right\}$ for $0 \leq \alpha+\beta \leq 4$ and $i=1,2,3$;

2) the derivatives $D_{\left\langle w_{i}, v\right\rangle}^{3} s\left(w_{i}\right)$ for $i=1,2,3$. 
Example 8.3. Any element in the space $\mathcal{S}_{14}^{6,9,9}\left(\triangle_{v}\right)$ of Theorem 4.1 with $m=3$ is uniquely defined by the following set of 172 data (cf. Example 4.3 and Figure 3):

1) the derivatives $\left\{D_{x}^{\alpha} D_{y}^{\beta} s\left(v_{i}\right)\right\}$ for $0 \leq \alpha+\beta \leq 9$ and $i=1,2,3$;

2) the derivatives $D_{\left\langle w_{i}, v\right\rangle}^{6} s\left(w_{i}\right)$ for $i=1,2,3$;

3) the derivatives $D_{\left\langle v_{i}, v\right\rangle}^{10} s\left(w_{i}\right)$ for $i=1,2,3$;

4) the value $s(v)$.

\section{Comparison with earlier Powell-Sabin macro-elements}

Macro-elements based on Powell-Sabin splits have been proposed in several earlier papers. For $r=2$, see [15], 21], [22], and for general $r$, see [8], 20, 24]. The following formulae (which can be easily verified using the above Bernstein-Bézier techniques) can be found in [24]:

$$
\begin{aligned}
\operatorname{dim} \mathcal{S}_{5 m}^{2 m, 3 m, 4 m-1}\left(\triangle_{P S}\right) & =\left(31 m^{2}+25 m+6\right) / 2, \\
\operatorname{dim} \mathcal{S}_{5 m+2}^{2 m+1,3 m+1,4 m+1}\left(\triangle_{P S}\right) & =\left(31 m^{2}+47 m+18\right) / 2 .
\end{aligned}
$$

Our macro-elements have two advantages over these macro-elements:

- they use lower degree polynomials,

- they use a smaller number of degrees of freedom,

for $r \geq 5$. Table 1 shows a comparison of the macro-elements in (9.1) with our new macro-elements for $1 \leq r \leq 10$. The columns $d$ and $n$ give the degree and number of degrees of freedom for our macro-elements, while the columns $\tilde{d}$ and $\tilde{n}$ give the same information for the earlier elements.

TABLE 1. Comparison of macro-elements

\begin{tabular}{|c|c|c|c|c|}
\hline$r$ & $d$ & $n$ & $\tilde{d}$ & $\tilde{n}$ \\
\hline 1 & 2 & 9 & 2 & 9 \\
2 & 5 & 31 & 5 & 31 \\
3 & 7 & 48 & 7 & 48 \\
4 & 10 & 90 & 10 & 90 \\
5 & 11 & 111 & 12 & 118 \\
6 & 14 & 172 & 15 & 180 \\
7 & 16 & 210 & 17 & 219 \\
8 & 19 & 291 & 20 & 301 \\
9 & 20 & 327 & 22 & 351 \\
10 & 23 & 427 & 25 & 453 \\
\hline
\end{tabular}

\section{Optimality of the MaCRO-Elements}

In this section we explore to what extent the macro-elements discussed here are optimal with respect to

1) stability of dimension,

2) degree of the splines,

3) number of degrees of freedom used,

for a given smoothness $r$. The best elements will have the lowest degree possible, will have stable dimensions, and will use the least number of degrees of freedom. 
We begin by examining the dimension of the superspline space

$$
\mathcal{S}:=\left\{s \in \mathcal{S}_{d}^{r}\left(\triangle_{v}\right): s \in C^{\rho_{v}}(v)\right\}
$$

defined on a Powell-Sabin cell. By Lemma 3.2 of [6],

$$
\operatorname{dim} \mathcal{S}=\left(\begin{array}{c}
\rho_{v}+2 \\
2
\end{array}\right)+6\left[\left(\begin{array}{c}
d-r+1 \\
2
\end{array}\right)-\left(\begin{array}{c}
\rho_{v}-r+1 \\
2
\end{array}\right)\right]+\sigma,
$$

where

$$
\sigma:=\sum_{j=\rho_{v}-r+1}^{d-r}(r+j+1-j e)_{+}
$$

and $e$ is the number of edges attached to the center vertex $v$ with different slopes. Since $3 \leq e \leq 6$ for a Powell-Sabin split, we conclude that a necessary condition for $\sigma$ to be zero for all configurations is that $\rho_{v}+2-3\left(\rho_{v}-r+1\right) \leq 0$, which is equivalent to

$$
\rho_{v} \geq\left\lceil\frac{3 r-1}{2}\right\rceil= \begin{cases}3 m, & \text { if } r=2 m, \\ 3 m+1, & \text { if } r=2 m+1 .\end{cases}
$$

We now show that in order for a Powell-Sabin macro-element to be capable of meshing with neighboring macro-elements without incompatibilities, we also have to enforce a certain supersmoothness at each of the vertices $v_{1}, v_{2}, v_{3}$.

Theorem 10.1. Suppose that $T:=\left\langle v_{1}, v_{2}, v_{3}\right\rangle$ is a triangle, and that $\triangle_{T}$ is a refinement such that there are $n \geq 0$ interior edges connected to the vertex $v_{1}$. Let $s$ be a spline of degree $d$ and smoothness $r$ defined on $\triangle_{T}$. Then the cross derivatives of $s$ up to order $r$ on the edges $e_{1}:=\left\langle v_{1}, v_{2}\right\rangle$ and $e_{2}:=\left\langle v_{1}, v_{3}\right\rangle$ can be specified independently only if we require $s \in C^{\rho}\left(v_{1}\right)$, with

$$
\rho \geq\left\lceil\frac{(n+2) r-n}{n+1}\right\rceil .
$$

Proof. We examine the ring $R_{\rho+1}\left(v_{1}\right)$ with $r \leq \rho$. The number of coefficients of $s$ on this ring is $(n+1)(\rho+1)+1$. Now setting cross-derivatives up to order $r$ on the edges $e_{1}$ and $e_{2}$ determines $2(r+1)$ of these coefficients. It follows that a necessary condition for the number of undetermined coefficients in these equations to be at least equal to the number of conditions is

$$
(n+1)(\rho+1)+1-2(r+1) \geq n r,
$$

or equivalently,

$$
\frac{(n+2) r-n}{n+1} \leq \rho .
$$

Thus, to avoid incompatible systems, we have to enforce supersmoothness of order $\rho$ at least equal to the integer (10.2).

Choosing $n=1$, it follows that to define macro-elements on the Powell-Sabin split of a triangle $T$, we must enforce supersmoothness of order

$$
\rho \geq\left\lceil\frac{3 r-1}{2}\right\rceil= \begin{cases}3 m, & \text { if } r=2 m, \\ 3 m+1, & \text { if } r=2 m+1\end{cases}
$$

at each vertex $v_{i}$ of $T$. 
We are now ready to show that the degrees of our Powell-Sabin macro-elements are minimal. Suppose

$$
s \in\left\{s \in \mathcal{S}_{d}^{r}\left(\triangle_{v}\right): s \in C^{\rho_{v}}(v) \text { and } s \in C^{\rho}\left(v_{i}\right) \text { for } i=1,2,3\right\}
$$

is a superspline defined on a Powell-Sabin cell which has supersmoothness $\rho$ at each of the vertices $v_{i}$ of the triangle $T$, and supersmoothness $\rho_{v}$ at the incenter $v$ of $\triangle_{v}$, where $\rho$ and $\rho_{v}$ satisfy (10.2) and (10.3). We now examine the coefficients of $s$ associated with domain points lying on the ring $R_{\rho_{v}}(v)$. In particular, we focus on the points in the $\left(d-\rho_{v}\right)$-th row parallel to the edge $\left\langle v_{1}, v_{2}\right\rangle$ of $T$. If we set the coefficients corresponding to the disks $D_{\rho}\left(v_{1}\right)$ and $D_{\rho}\left(v_{2}\right)$, then there remain $2 \rho_{v}+1-2\left(\rho+\rho_{v}-d+1\right)$ unset coefficients in the middle of this row. They must satisfy $\rho_{v}$ conditions for $C^{\rho_{v}}$ continuity across the edge $\left\langle v, w_{1}\right\rangle$. In general this will lead to an incompatibility unless the number of unset coefficients is at least $\rho_{v}$. It follows that such macro-elements exist only if

$$
d \geq\left\lceil\frac{\rho_{v}+2 \rho+1}{2}\right\rceil .
$$

Now inserting the minimal possible value (10.3) for both $\rho$ and $\rho_{v}$ and examining the resulting four cases corresponding to $r=2 k$ and $r=2 k+1$ with $k$ both even and odd, we see that the dimensions of our Powell-Sabin macro-elements are indeed minimal.

Finally, we briefly discuss the question of degrees of freedom. By the above, we know that the minimal determining set for any Powell-Sabin macro-element must include the disks $D_{\rho}\left(v_{i}\right)$ for $i=1,2,3$, where $\rho$ satisfies (10.3). This means that the lowest possible number of degrees of freedom for such an element is

$$
n_{m}:=\left\{\begin{array}{cl}
3\left(\begin{array}{c}
3 m+2 \\
2
\end{array}\right)=\frac{27 m^{2}+27 m+6}{2}, & r=2 m, \\
3\left(\begin{array}{c}
3 m+3 \\
2
\end{array}\right)=\frac{27 m^{2}+45 m+18}{2}, & r=2 m+1 .
\end{array}\right.
$$

These numbers are only slightly smaller than the dimensions of our Powell-Sabin macro-elements. There are two ways in which the number of degrees of freedom of our elements could be lowered:

1) increase $\rho_{v}$ (subject to (10.4));

2) enforce some additional smoothness across certain interior edges of $\triangle_{v}$.

We have examined various possible modifications of this type to our macroelements, but so far have not found any elements with fewer degrees of freedom.

\section{REMARKS}

Remark 11.1. Powell-Sabin splits were introduced in [19].

Remark 11.2. The degrees of the spline spaces dealt with in this paper are substantially smaller than $3 r+2$, as shown in Table 2 .

Remark 11.3. It was shown in Section 10 of [16] that if a space of splines $\mathcal{S}$ of degree $d$ contains $\mathcal{P}_{d}$ and has a stable local basis, then it provides optimal order approximations of smooth functions. In particular, for every $0 \leq k \leq d$, there exists a quasi-interpolation operator $Q_{k}$ such that for every function $f \in W_{p}^{k+1}(\Omega)$,

$$
\left\|D_{x}^{\alpha} D_{y}^{\beta}\left(f-Q_{k} f\right)\right\|_{p} \leq K|\triangle|^{k+1-\alpha-\beta}|f|_{k+1, p}
$$

for $0 \leq \alpha+\beta \leq k$, where $|\triangle|$ is the mesh size of $\triangle$ (i.e., the diameter of the largest triangle), and $|f|_{k+1, p}$ is the usual Sobolev semi-norm. If $\Omega$ is convex, then 
TABle 2. Comparison of $d(r)$ with $3 r+2$

\begin{tabular}{|c|c|c|c|}
\hline$m$ & $r$ & $d(r)$ & $3 r+2$ \\
\hline 0 & 1 & 2 & 5 \\
1 & 2 & 5 & 8 \\
1 & 3 & 7 & 11 \\
2 & 4 & 10 & 14 \\
2 & 5 & 11 & 17 \\
3 & 6 & 14 & 20 \\
3 & 7 & 16 & 23 \\
4 & 8 & 19 & 26 \\
4 & 9 & 20 & 29 \\
\hline
\end{tabular}

the constant $K$ depends only on $d, p, k$, and on the smallest angle $\theta_{\triangle}$ in $\triangle$. If $\Omega$ is nonconvex, it also depends on the Lipschitz constant $L_{\partial \Omega}$ associated with the boundary of $\Omega$. In view of our construction of stable local bases for the spaces $\mathcal{S}_{m}\left(\triangle_{P S}\right)$ in Sections 4-7 for general $m \geq 0$, we can conclude that all of these spaces have full approximation power.

Remark 11.4. For $d<3 r+2$, it is known [7] that the spaces $\mathcal{S}_{d}^{r}(\triangle)$ do not possess optimal order approximation order for arbitrary triangulations. This means that neither they (nor any subspace $\mathcal{S}$ containing $\mathcal{P}_{d}$ ) has a stable local basis.

Remark 11.5. Macro-elements and stable local bases can be constructed for several other refinement methods. In [18] we do this for the well-known Clough-Tocher split. For results on quadrangulations, see [14], 17].

Remark 11.6. For special results on Powell-Sabin elements associated with uniform three-direction (type-I) meshes, see [9]-[12].

\section{ACKNOWLEDGMENTS}

We would like to thank Peter Alfeld for writing a beautiful JAVA program which computes dimensions of spline spaces (in exact arithmetic) and which can also be used for verifying minimal determining sets. We have made extensive use of this software in preparing this paper and checking our results. It can be accessed at http://www.math.utah/ alfeld/MDS/index.html.

\section{REFERENCES}

1. Alfeld, P., Schumaker, L. L., The dimension of bivariate spline spaces of smoothness $r$ for degree $d \geq 4 r+1$, Constr. Approx., 3 (1987), 189-197. MR 88e:41025

2. Chui, C. K., Hong, D., Jia, R. Q., Stability of optimal-order approximation by bivariate splines over arbitrary triangulations, Trans. Amer. Math. Soc., 347(1995), 3301-3318. MR 96d:41012

3. Davydov, O., Schumaker, L. L., On stable local bases for bivariate polynomial splines, Constr. Approx., to appear.

4. Farin, G., Curves and Surfaces for Computer Aided Geometric Design, Academic Press (NY), 1988. MR 90c:65014

5. Hoschek, J., Lasser, D., Fundamentals of Computer Aided Geometric Design, A. K. Peters (Boston MA), 1993. MR 94i:65003

6. Ibrahim, A., Schumaker, L. L., Super spline spaces of smoothness $r$ and degree $d \geq 3 r+2$, Constr. Approx., 7(1991), 401-423. MR 92k:41017 
7. Jia, R. Q., Approximation order from certain spaces of smooth bivariate splines on a threedirection mesh, Trans. Amer. Math. Soc., 295(1986), 199-212. MR 88d:41018

8. Laghchim-Lahlou, M., Eléments finis composites de classe $C^{k}$ dans $\mathbb{R}^{2}$, Thèse de Doctorat, INSA de Rennes, 1991.

9. Laghchim-Lahlou, M., Composite $C^{r}$-triangular finite elements of PS type on a three direction mesh, (Curves and Surfaces), P.-J. Laurent, A. Le Méhauté, and L. L. Schumaker (eds.), Vanderbilt Univ. Press (Nashville), 1991, 275-278. CMP 91:17

10. Laghchim-Lahlou, M., $C^{r}$-finite elements of Powell-Sabin type on the three direction mesh, Advances in Comp. Math., 6(1996), 191-206. MR 97j:65031

11. Laghchim-Lahlou, M., The $C^{r}$-fundamental splines of Clough-Tocher and Powell-Sabin types for Lagrange interpolation on a three-direction mesh, Advances in Comp. Math., 8(1998), 353-366. MR 99j:65015

12. Laghchim-Lahlou, M., Interpolation d'Hermite ou de Lagrange dans des espaces de supersplines composites dan le plan, Thesis, Univ. Cadi Ayyad, Marrakesh, 1998.

13. Laghchim-Lahlou, M., Sablonnière, P., Triangular finite elements of HCT type and class $C^{\rho}$, Advances in Comp. Math., 2(1994), 101-122. MR 95d:65013

14. Laghchim-Lahlou, M., Sablonnière, P., Quadrilateral finite elements of FVS type and class $C^{\rho}$, Numer. Math., 70(1995), 229-243. MR 96e:65006

15. Lai, M.-J., On $C^{2}$ quintic spline functions over triangulations of Powell-Sabin's type, J. Comput. Appl. Math., 73(1996), 135-155. MR 98a:41002

16. Lai, M.-J., Schumaker, L. L., On the approximation power of bivariate splines, Advances in Comp. Math., 9(1998), 251-279. MR 2000b:41010

17. Lai, M.-J., Schumaker, L. L., On the approximation power of splines on triangulated quadrangulations, SIAM J. Numer. Anal., 36(1999), 143-159. MR 99h:41014

18. Lai, M.-J., Schumaker, L. L., Macro-elements and stable local bases for spaces of splines on Clough-Tocher triangulations, Numerische Math., to appear.

19. Powell, M. J. D., Sabin, M. A., Piecewise quadratic approximations on triangles, ACM Trans. Math. Software, 3(1977), 316-325. MR 58:3319]

20. Sablonnière, P., Composite finite elements of class $C^{k}$, J. Comp. Appl. Math., 12(1985), 541-550. CMP 17:14

21. Sablonnière, P., Eléments finis triangulaires de degré 5 et de classe $C^{2}$, (Computers and Computing), P. Chenin et al (eds.), Wiley (New York), 1986, 111-115.

22. Sablonnière, P., Composite finite elements of class $C^{2}$, Topics in Multivariate Approximation, C. K. Chui, L. L. Schumaker, and F. Utreras (eds.), Academic Press (New York), 1987, 207-217. MR 90b:65212

23. Sablonnière, P., Error bounds for Hermite interpolation by quadratic splines on an $\alpha$ triangulation, IMA J. Numer. Anal., 7(1987), 495-508. MR 90a:65029

24. Sablonnière, P., Laghchim-Lahlou, M., Eléments finis polynomiaux composés de classe $C^{r}, \mathrm{C}$. R. Acad. Sci. Paris Sr. I Math., 316(1993), 503-508. MR 94a:65059

Department of Mathematics, The University of Georgia, Athens, Georgia 30602

E-mail address: mjlai@math.uga.edu

Department of Mathematics, Vanderbilt University, Nashville, Tennessee 37240

E-mail address: s@mars.cas.vanderbilt.edu 